\title{
Toward Rapid and Well-controlled Ambient Temperature RAFT Polymerization under UV-vis Radiation: Effect of Radiation Wave Range
}

\author{
Lican Lu, Haijia Zhang, Nianfa Yang and Yuanli Cai*
}

College of Chemistry, Xiangtan University, Xiangtan, Hunan 411105, P. R. China

\section{Supporting Information}




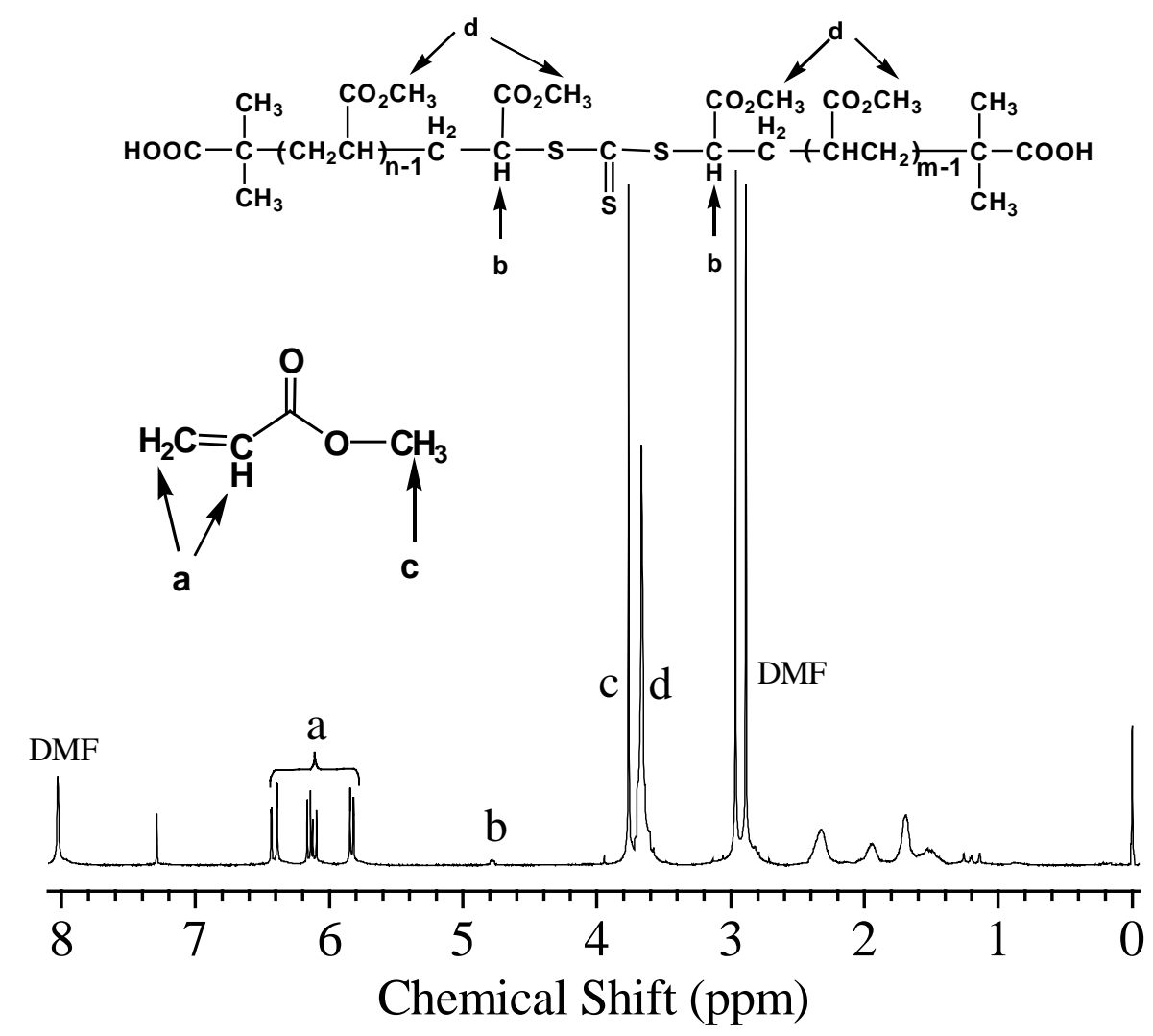

Figure S1 ${ }^{1} \mathrm{H}$ NMR spectrum of methyl acrylate (MA) polymerizing solution under long-wave radiation predominantly at $365-405 \mathrm{~nm}$ with intensity of $100 \mu \mathrm{W} \mathrm{cm}{ }^{-2}$ at $365 \mathrm{~nm}$ and $80 \mu \mathrm{W}$ $\mathrm{cm}^{-2}$ at $420 \mathrm{~nm} ;[\text { BDMAT }]_{0}=56.3 \mathrm{mmol} \mathrm{L}^{-1},[\mathrm{MA}]_{0}:[\mathrm{BDMAT}]_{0}:[\mathrm{TPO}]_{0}=150: 1: 0.1 \mathrm{in}$ $N, N$-dimethylformamide at $30{ }^{\circ} \mathrm{C}$ for $2 \mathrm{~h}$. BDMAT, $S, S$ '-bis $\left(\alpha, \alpha^{\prime}\right.$-dimethyl- $\alpha$ "-acetic acid) trithiocarbonate; TPO, (2,4,6-trimethylbenzoyl) diphenylphosphine oxide. 


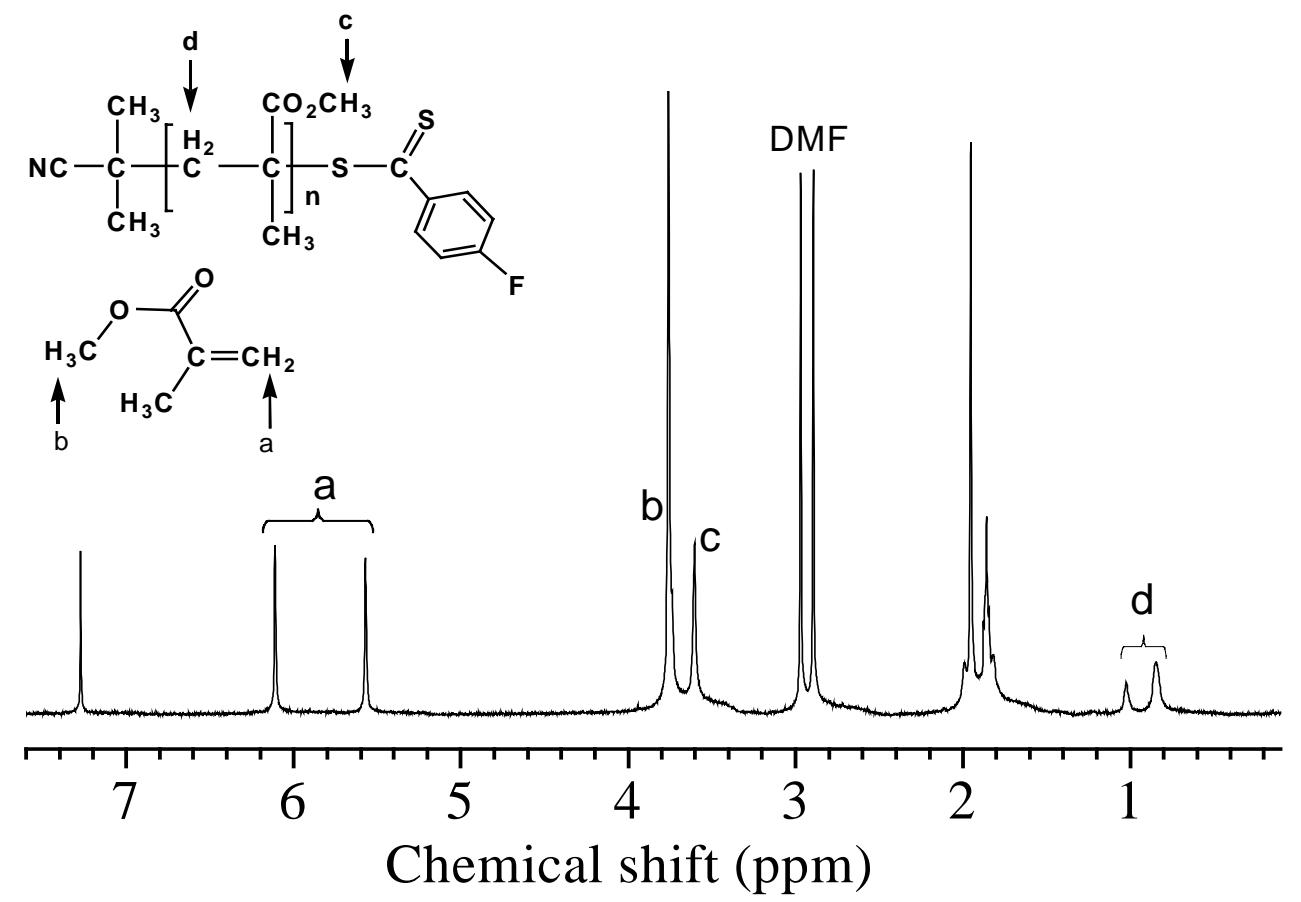

Figure S2 ${ }^{1} \mathrm{H}$ NMR spectrum of methyl methacrylate (MMA) polymerizing solution under visible radiation in the wave range above $380 \mathrm{~nm}$ predominantly at $405 \mathrm{~nm}$ with intensity of $0.8 \mu \mathrm{W} \mathrm{cm} \mathrm{cm}^{-2}$ at $365 \mathrm{~nm}$ and $420 \mu \mathrm{W} \mathrm{cm}{ }^{-2}$ at $420 \mathrm{~nm}$; [CPFDB] $]_{0}=24.0 \mathrm{mmol} \mathrm{L}^{-1}$, $[\mathrm{MMA}]_{0}:[\mathrm{CPFDB}]_{0}:[\mathrm{TPO}]_{0}=300: 1: 0.4$ in $N, N$-dimethylformamide at $30{ }^{\circ} \mathrm{C} . \mathrm{CPFDB}$, 2-cyanoprop-2-yl(4-fluoro) dithiobenzoate; TPO, (2,4,6-trimethylbenzoyl) diphenylphosphine oxide. 


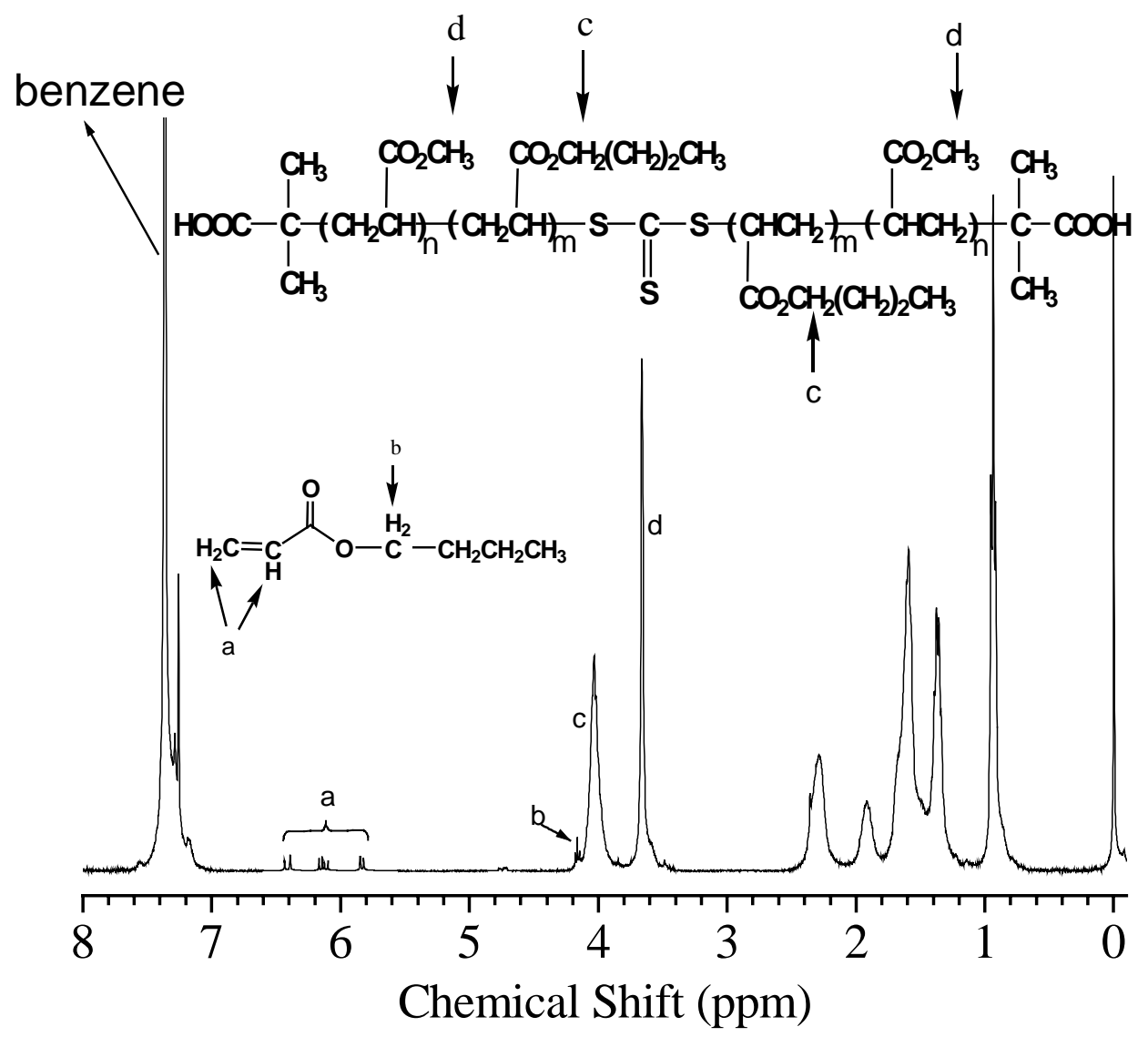

Figure S3 ${ }^{1} \mathrm{H}$ NMR spectrum of the block copolymerizing solution. Conditions: poly(methyl acrylate) (PMA, ${ }^{1} \mathrm{H}$ NMR: $92 \%$ conversion; UV-vis spectrophotometry: 5\% of photolysis degree; GPC: $\left.\mathrm{M}_{\mathrm{n}}=11400, \mathrm{M}_{\mathrm{w}} / \mathrm{M}_{\mathrm{n}}=1.10\right)$ as a macro-CTA; under long-wave radiation predominantly at $365-405 \mathrm{~nm}$ with intensity of $60 \mu \mathrm{W} \mathrm{cm}{ }^{-2}$ at $365 \mathrm{~nm}$ and $49 \mu \mathrm{W} \mathrm{cm}^{-2}$ at 420 $\mathrm{nm} ;[n \mathrm{BA}]_{0}:[\mathrm{PMA}]_{0}:[\mathrm{TPO}]_{0}=200: 1: 0.1$ in $50 \mathrm{v} . \%$ benzene at $30{ }^{\circ} \mathrm{C}$ for $4 \mathrm{~h} . \mathrm{TPO}$, (2,4,6-trimethylbenzoyl) diphenylphosphine oxide; $n \mathrm{BA}, n$-butyl acrylate. 


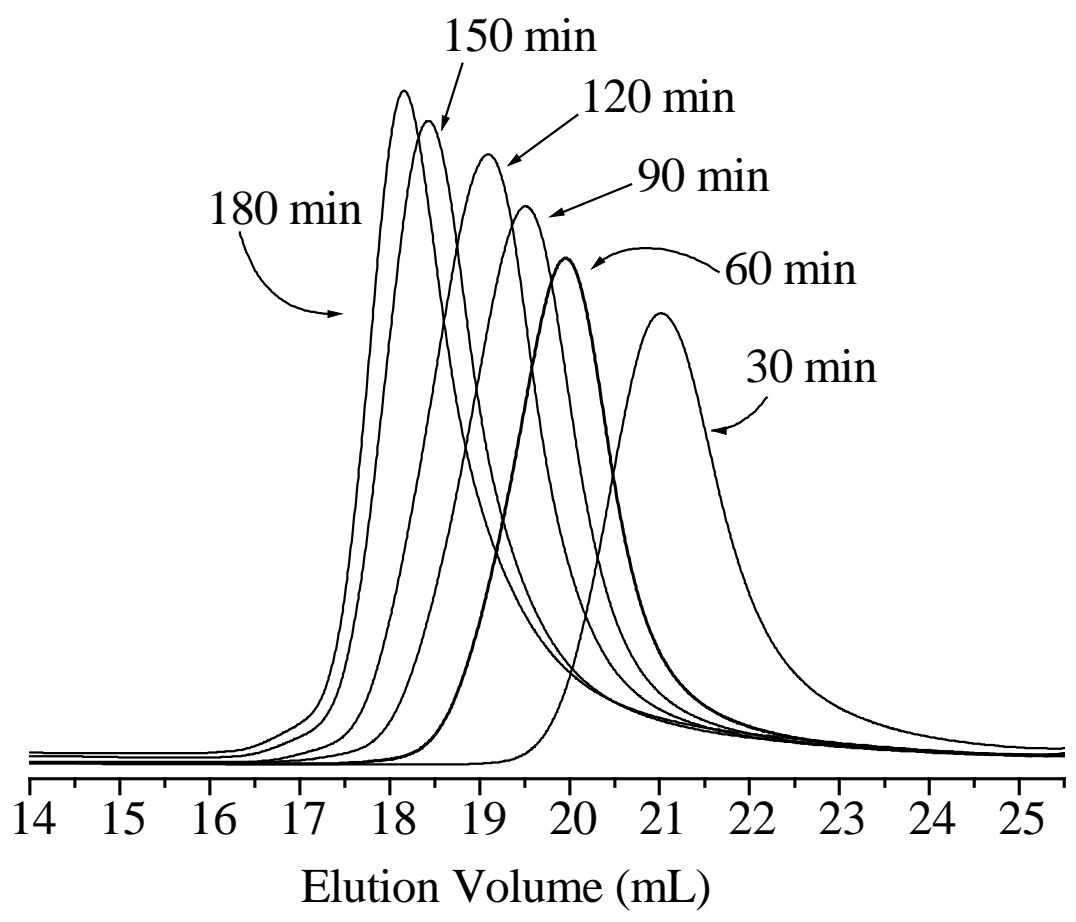

Figure S4 GPC traces of poly(methyl acrylate) (PMA) obtained from the RAFT polymerization of methyl acrylate (MA) under long-wave radiation with intensity of $100 \mu \mathrm{W}$ $\mathrm{cm}^{-2}$ at $365 \mathrm{~nm}$ and $80 \mu \mathrm{W} \mathrm{cm}$ at $420 \mathrm{~nm} ; \quad[\text { BDMAT] }]_{0}=56.3 \mathrm{mmol} \mathrm{L} \mathrm{L}^{-1}$, $[\mathrm{MA}]_{0}:[\mathrm{BDMAT}]_{0}:[\mathrm{TPO}]_{0}=150: 1: 0.1$ in $N, N$-dimethylformamide at $30{ }^{\circ} \mathrm{C}$. BDMAT, $S, S^{\prime}$-bis $\left(\alpha, \alpha^{\prime}\right.$-dimethyl- $\alpha$ "-acetic acid $) \quad$ trithiocarbonate; TPO, (2,4,6-trimethylbenzoyl) diphenylphosphine oxide. 